Check for updates

Division of Infectious Diseases, University of Pennsylvania, Philadelphia, PA, USA

2 Division of Infectious Diseases and Geographic Medicine, School of Medicine, Stanford University, Palo Alto, CA, USA

3 Division of Infection and Global Health, School of Medicine, University of St Andrews, St Andrews, UK

Correspondence to: M Cevikmc349@standrews.ac.uk

https://orcid.org/0000-0003-1133-3874

Cite this as: BMJ 2021;375:n3105

http://dx.doi.org/10.1136/bmj.n3105

Published: 20 December 2021

\section{Covid-19 vaccines, immunity, and boosters}

\author{
Many people still lack essential (and enduring) protection from primary vaccination
}

\author{
Aaron Richterman, ${ }^{1}$ Jake Scott, ${ }^{2}$ Muge Cevik ${ }^{3}$
}

Since our previous editorial in September 2021, ${ }^{1} \mathrm{a}$ growing body of evidence about covid-19 vaccine effectiveness, including that of third and booster doses, has been published, and several countries have authorized boosters for general adult populations. $^{2}$

Well conducted real world studies of vaccine effectiveness are an important complement to randomized controlled trials. For example, two linked studies use test negative designs to analyze rich datasets from large health systems. Firstly, Israel and colleagues (doi:10.1136/bmj-2021-067873) estimated changes over time in the effectiveness of the Pfizer-BioNTech BNT162b2 vaccine against SARS-CoV-2 infection among members of a nationwide healthcare system in Israel during a period dominated by the delta variant. ${ }^{3}$ They found an increased risk of infection associated with intervals longer than three months since full vaccination. Adjusted odds ratios were 2.37 at three to four months after vaccination but increased only slightly to 2.82 at six months or more.

The second study, by Bruxvoort and colleagues (doi:10.1136/bmj-2021-068848), evaluated the effectiveness of Moderna's mRNA-1273 vaccine against SARS-CoV-2 variants including delta, alpha, $\mathrm{mu}$, and others among 8153 cases and matched controls in an integrated healthcare system in California. ${ }^{4}$ Vaccine effectiveness against infection with the delta variant was $94.1 \%$ (95\% confidence interval $90.5 \%$ to $96.3 \%$ ) at two months or less after vaccination, declining to $80.0 \%(70.2 \%$ to $86.6 \%)$ at five to six months. For non-delta variants, vaccine effectiveness was higher and more stable over time, declining from $98.6 \%$ at two months or less to $88.7 \%$ at five to six months. Importantly, vaccine effectiveness against admission to hospital with the delta variant remained at $97.5 \%$ (92.7\% to $99.2 \%)$.

Although these studies provide valuable insights, all observational studies are vulnerable to biases related to underlying differences between the studied populations, which can lead to differences between the estimated and true effectiveness. For context, consider the initial randomized trial evaluating the BNT162b2 vaccine, conducted before the emergence of the delta variant, which reported an estimated efficacy against symptomatic infection by pre-delta variants of $96.2 \%$ (93.3\% to $98.1 \%)$ at two months or less, $90.1 \%(86.6 \%$ to $92.9 \%)$ at two to four months, and $83.7 \%(74.7 \%$ to $89.9 \%)$ at four to six months after vaccination. ${ }^{5}$ These changes over time are consistent with Israel and colleagues' findings, indicating that residual bias in that study is likely small. We can have confidence in their observation that effectiveness remains relatively stable beyond six months, even in the context of delta. Supporting this, in a post hoc analysis during a period dominated by delta, differences in infection rates between participants originally randomized to the vaccine and those who received the vaccine after unblinding six months later suggest a minimal and more gradual decline in efficacy from $83.7 \%$ at four to six months to $78 \%$ at $10-12$ months. ${ }^{6}$ Together, the observational and randomized data to date suggest that after an initial decline, protection may become more stable, even in the context of delta.

We previously argued that studies showing modest waning of immunity do not support indiscriminate use of booster doses outside of older and medically vulnerable populations. ${ }^{7}$ A randomized controlled trial (not yet peer reviewed) has since found that a third dose of BNT162b2, about 11 months after the primary course, gave $95.3 \%$ (88.5\% to $97.9 \%)$ relative efficacy against symptomatic infection during 2.5 months of follow-up, compared with two doses alone. ${ }^{8}$ This is consistent with observational data of booster effectiveness, ${ }^{9-11}$ including a rigorously conducted matched cohort study showing that these benefits extend to severe outcomes, primarily among older people. ${ }^{12}$ Booster doses may also have a role in helping to reduce transmission in well vaccinated populations during periods of substantial community transmission. ${ }^{13}$

Research still in preprint suggests that the new omicron variant is associated with reduced neutralizing antibody responses following two doses of vaccine, which is reversed by a booster dose or hybrid immunity from a combination of vaccination and infection. ${ }^{1415}$ A reduction in vaccine effectiveness and improved protection afforded by booster doses is also supported by preliminary clinical data from the UK. ${ }^{16}$ That broader and increased antibody titers generated by a third or booster dose may be able to overcome the reduced neutralization associated with the omicron variant is therefore plausible. Further research evaluating the effectiveness of primary and additional vaccine doses against omicron is clearly a priority.

Although a third or booster dose clearly provides additional protection on top of simply reversing previous waning, the greatest protection from the worst clinical outcomes remains heavily concentrated in the first two doses. ${ }^{17}$ The long term durability of protection against hospital admission afforded by two dose vaccine regimens is clear, particularly with an extended interval between the two doses (and even in the face of new variants). ${ }^{18}$ Given the importance of reducing disease burden globally, vaccinations in low income settings, where the vast majority of people are yet to receive even a first dose, 
should be prioritized over additional doses in high income settings. Policies that preferentially stockpile vaccine doses in high income settings remain indefensible. Although we do not know the precise circumstances that led to the emergence of omicron, that the extreme disparities in access to vaccines between high income and low income settings create the ideal conditions for the ongoing evolution of SARS-CoV-2 is clear.

Competing interests: I have read and understood the BMJ Group policy on declaration of interests and declare the following interests: none.

The BMJ policy on financial interests is here: https://www.bmj.com/sites/default/files/attachments/resources/2016/03/16-current-bmj-education-coi-form.pdf.

Provenance and peer review: Commissioned; not peer reviewed.

1 Scott J, Richterman A, Cevik M. Covid-19 vaccination: evidence of waning immunity is overstated BMJ2021;374:n2320. doi: 10.1136/bmj.n2320 pmid: 34556464

2 Parker C, Pietsch B. Countries around the world are debating coronavirus booster shots. Here's where they've been approved. 2021. https://www.washingtonpost.com/world/2021/11/12/coronavirus-vaccine-boosters-global/.

3 Israel A, Merzon E, Schäffer AA, etal. Elapsed time since BNT162b2 vaccine and risk of SARS-CoV-2 infection: test negative design study. BMJ 2021;375:e067873. doi: 10.1136/bmj-2021-067873 pmid: 34819275

4 Bruxvoort KJ, Sy LS, Qian L, etal. Effectiveness of mRNA-1273 against delta, mu, and other emerging variants of SARS-CoV-2: test negative case-control study. BMJ 2021;375:e068848. doi: 10.1136/bmj-2021-068848 pmid: 34911691

5 Thomas SJ, Moreira ED, JrKitchin N, etalC4591001 Clinical Trial Group. Safety and Efficacy of the BNT162b2 mRNA Covid-19 Vaccine through 6 Months. N Engl J Med 2021;385:1761-73. doi: 10.1056/NEJMoa2110345 pmid: 34525277

6 US Food and Drug Administration. Application for licensure of a booster dose for COMIRNATY (COVID-19 Vaccine, mRNA). 2021. https://www.fda.gov/media/152176/download.

7 Andrews N, Tessier E, Stowe J, etal. Vaccine effectiveness and duration of protection of Comirnaty, Vaxzevria and Spikevax against mild and severe COVID-19 in the UK.medRxiv 2021:2021.09.15.21263583doi: 10.1101/2021.09.15.21263583.

8 Perez JL. Efficacy \& Safety of BNT162b2 booster - C4591031 2-month interim analysis. 2021. https://www.cdc.gov/vaccines/acip/meetings/downloads/slides-2021-11-19/02-COVID-Perez508.pdf.

9 Bar-On YM, Goldberg Y, Mandel M, etal. Protection of BNT162b2 vaccine booster against covid-19 in Israel. N Engl J Med 2021;385:1393-400. doi: 10.1056/NEJMoa2114255 pmid: 34525275

10 Bar-On YM, Goldberg Y, Mandel M, etal. Protection against Covid-19 by BNT162b2 booster across age groups. N Engl J Med 2021. doi: 10.1056/NEJMoa2114255 pmid: 34879188

11 Arbel R, Hammerman A, Sergienko R, etal. BNT162b2 vaccine booster and mortality due to Covid-19. N Engl J Med 2021. doi: 10.1056/NEJMoa2115624 pmid: 34879190

12 Barda N, Dagan N, Cohen C, etal. Effectiveness of a third dose of the BNT162b2 mRNA COVID-19 vaccine for preventing severe outcomes in Israel: an observational study. Lancet 2021;398:2093-100. doi: 10.1016/S0140-6736(21)02249-2 pmid: 34756184

13 Meyerowitz E, Richterman A. SARS-CoV-2 Transmission and Prevention in the Era of the Delta Variant. 2021. https://papers.ssrn.com/sol3/papers.cfm?abstract_id=3964247.

14 Cele S, Jackson L, Khan K, etal. SARS-CoV-2 Omicron has extensive but incomplete escape of Pfizer BNT162b2 elicited neutralization and requires ACE2 for infection.medRxiv 2021:2021.12.08.21267417doi: 10.1101/2021.12.08.21267417

15 Schmidt F, Muecksch F, Weisblum Y, etal. Plasma neutralization properties of the SARS-CoV-2 Omicron variantMedRxiv 2021:2021.12.12.21267646doi: 10.1101/2021.12.12.21267646.

16 Andrews N, Stowe J, Kirsebom F, etal. Effectiveness of COVID-19 vaccines against the Omicron (B.1.1.529) variant of concern. medRxiv 2021:2021.12.14.21267615doi: 10.1101/2021.12.14.21267615

17 Cevik M, Grubaugh ND, Iwasaki A, Openshaw P. COVID-19 vaccines: Keeping pace with SARS-CoV-2 variants. Cell 2021;184:5077-81. doi: 10.1016/j.cell.2021.09.010 pmid: 34534444

18 Skowronski DM, Setayeshgar S, Febriani Y, etal. Two-dose SARS-CoV-2 vaccine effectiveness with mixed schedules and extended dosing intervals: test-negative design studies from British Columbia and Quebec, Canada.medRxiv

2021:2021.10.26.21265397doi: 10.1101/2021.10.26.21265397

This article is made freely available for use in accordance with BMJ's website terms and conditions for the duration of the covid-19 pandemic or until otherwise determined by BMJ. You may use, download and print the article for any lawful, non-commercial purpose (including text and data mining) provided that all copyright notices and trade marks are retained. 